\title{
URBAN AREA MONITORING VIA SYNERGIC USE OF COSMO-SKYMED AND RADARSAT-2 DATA
}

\author{
Gerardo Di Martino, Antonio Iodice, Daniele Riccio, Giuseppe Ruello \\ Università di Napoli Federico II, Via Claudio 21, 80125, Napoli, Italy
}

\begin{abstract}
In this paper we describe preliminary results obtained in the framework of a project selected in the context of the joint Announcement of Opportunity for the synergic use of Cosmo-SkyMed and RADARSAT-2 data. The project involves the analysis of SAR images of urban areas and, in particular, the synergic use of Cosmo/SkyMed and RADARSAT-2 data.
\end{abstract}

Index Terms - Synthetic Aperture Radar, urban areas

\section{INTRODUCTION}

The interpretation of microwave high-resolution SAR amplitude images of urban areas is hampered by the presence of multiple reflection contributions, due to dihedral and trihedral structures formed by buildings and ground and/or to the electromagnetic interaction among the different buildings present on the scene. For this reason, an effective analysis of SAR images of urban areas should be based on the use of adequate electromagnetic models able to account for the radiometric and geometric aspects involved in SAR image formation mechanisms: in particular, the radiometric aspects provide the possibility to relate relevant scene's physical quantities (e.g. dielectric constants, objects dimension and roughness) with the measured backscattered intensity, while the geometric ones refer to the evaluation of the final position of the single objects' contributions in the image plane. Actually, papers [1] and [2] go in the outlined direction, allowing a sound interpretation of many phenomena from both a radiometric and a geometric viewpoint [3], whereas approaches based on ray-tracing concepts frequently allow for a more flexible understanding of image geometrical aspects, at the cost of a significantly less accurate radiometric modeling [4], [5]. Anyway, in both cases the use of models, possibly allowing the implementation of simulation techniques, is of key importance for SAR image interpretation.

In this paper we present the preliminary results obtained in the framework of the project "Buildings Feature Extraction from Single SAR Images - Joint Application to COSMOSkyMed - RADARSAT-2 Images" selected in the context of the joint Announcement of Opportunity for the synergic use of Cosmo-SkyMed and RADARSAT-2 data. In particular, we discuss here the potentiality of urban areas information extraction based on the multi-frequency capabilities of the Cosmo-SkyMed/RADARSAT-2 joint system. At difference with most state-of-the-art contributions present in the scientific literature regarding the applications of multifrequency SAR systems, which are strongly data-dependent (in the sense that the physical interpretation phase typically follows the data-fusion step), the proposed approach is based on the preliminary development of analytical models for the synergic use of the dual-frequency CosmoSkyMed/RADARSAT-2 configuration. In fact, these two sensors share very similar parameters, differing mainly in operative frequencies ( $\mathrm{X}$ and $\mathrm{C}$ band, respectively). The proposed models are derived from the models in [1] upon evaluation at $\mathrm{X}$ and $\mathrm{C}$ band and they allow gaining a deeper insight on the specific characteristics of the two sensors, useful for the development of new techniques for the analysis of SAR images and for the extraction of value-added information.

\section{SCATTERING MODELS}

The development of the proposed models starts from the observation that the models presented in [1] are explicitly frequency dependent. Actually, from an electromagnetic viewpoint, this kind of dependence is rather unsurprising, because the backscattered signal intensity is always dependent on the relative weight of the object (i.e. soil or man-made target) roughness with respect to the electromagnetic wavelength $\lambda$. The soil roughness is here modeled as a stationary Gaussian process with standard deviation $\sigma$ and correlation length $L$, and Geometrical Optics (GO) and Physical Optics (PO) solutions are used to evaluate the different contributions (in particular, single, double and triple reflections are considered) of the field scattered by a single isolated building (modeled as a parallelepiped with smooth walls) placed on the rough terrain. Under these hypotheses, the Normalized Radar Cross Section (NRCS) $\sigma^{o}$ is a function of both sensor and scene parameters:

$\sigma^{o}=f\left(h, l, \sigma, L, \varepsilon_{w}, \varepsilon_{s}, \varphi, \vartheta, p q, k\right)$

where $h$ is the building height and $l$ its width, $\varepsilon_{w}$ and $\varepsilon_{s}$ are the complex dielectric constants of walls and soil respectively, $\varphi$ is the angle formed by the building façade 
with respect to the sensor's line of sight, $\vartheta$ is the incidence angle, $p q$ is the considered polarization ( $p$ and $q$ stand for $\mathrm{H}$ or $\mathrm{V}$ polarization), and, finally, $k=2 \pi / \lambda$ is the electromagnetic wavenumber. Note that through the parameter $l$ the NRCS also depends on sensor resolution [2]: in fact, for high-resolution sensors, the building is not imaged within a single resolution cell and $l$ represents the length of the portion of building façade enclosed in a single cell.

For instance, the expression of the double reflection contribution for the GO-PO case is

$$
\begin{array}{r}
\sigma^{o}=\left|S_{p q}\right|^{2} h l \tan \vartheta \cos \varphi \exp \left(-4 k^{2} \sigma^{2} \cos ^{2} \vartheta\right) \\
\sum_{m=1}^{\infty} \frac{(2 k \sigma \cos \vartheta)^{2 m}}{m !} \frac{k^{2} L^{2}}{4 m} \exp \left[-\frac{(2 k L \sin \varphi \sin \vartheta)^{2}}{4 m}\right]
\end{array}
$$

where $S_{p q}$ is the generic element of the scattering matrix for the $p q$-polarized case, depending on $\vartheta, \varphi, \varepsilon_{w}$ and $\varepsilon_{s}$. It is important to note that $\varepsilon_{w}$ and $\varepsilon_{s}$ depend on the frequency. This contribution will be located at the base of the building in the image plane [2]. Assuming that the orbits and the look angles of Cosmo-SkyMed and RADARSAT-2 acquisitions of the selected area are the same, after proper absolute calibration, the joint model applied to the same building defines the specific dependence on $k$ (i.e. in wavelength) and $l$ (i.e. resolution). Finally, if, in addition, comparable resolutions can be obtained, the joint model is uniquely dependent on the wavelength.

It is important to note that the introduced models assume that the considered buildings are electromagnetically isolated from each other: in actual scenarios this is frequently not the case. In more involved situations, contributions related to multiple reflections involving more than one building appear. In some cases, also the positions and the intensities of these contributions can be evaluated via adequate modifications of the same introduced models [6].

\section{DATA ACQUISITION}

The first joint acquisition campaign of Cosmo-SkyMed and RADARSART-2 data has been planned over the city of Naples, Italy, according to the discussion reported in Section 2. First data will be available from June 2015. The main requirements considered for the acquisition phase are:

- similar orbit and incidence angle

- short time interval between the acquisitions of the two sensors

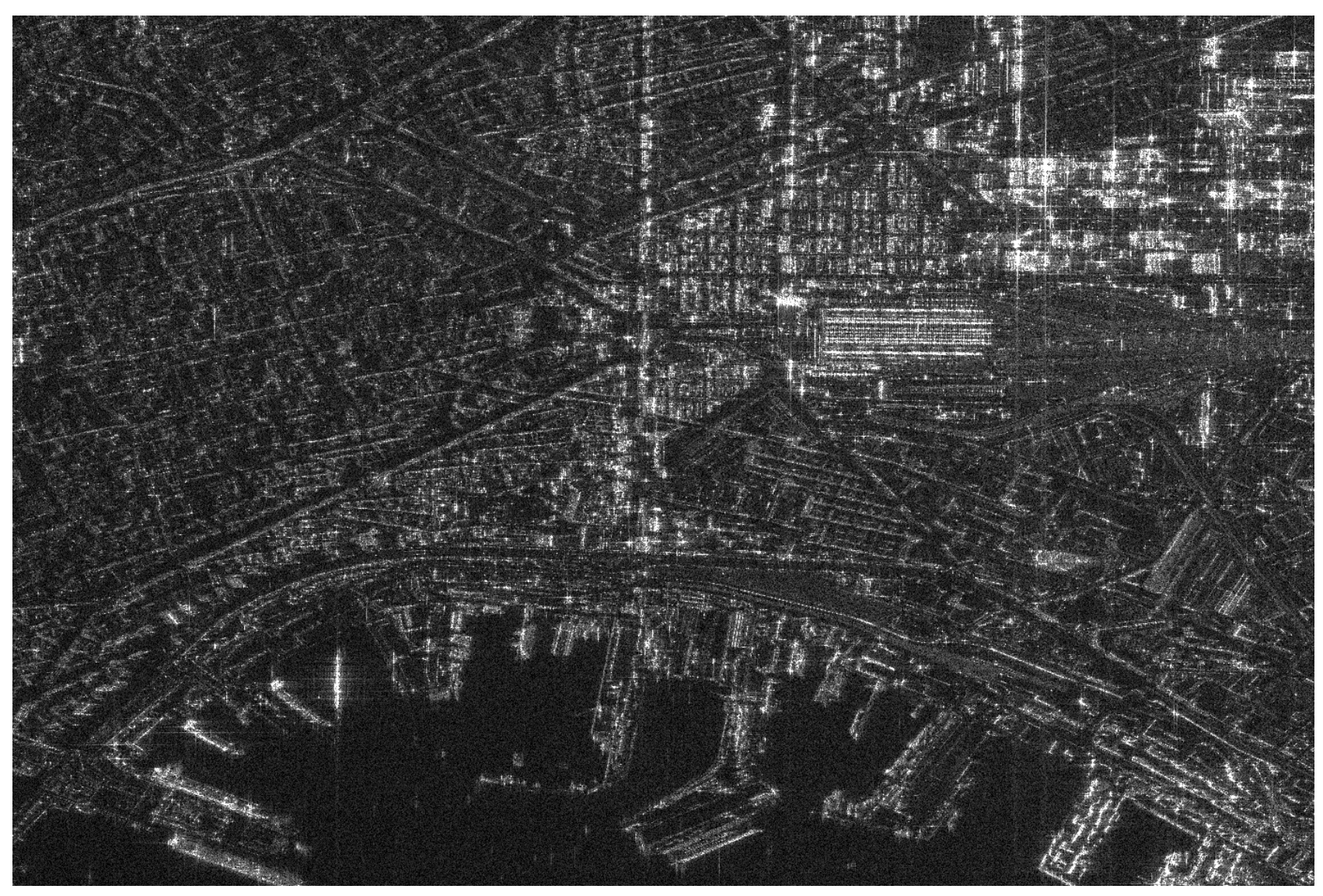

Fig. 1 Subset of the COSMO/SkyMed enhanced spotlight image of the city of Naples: the look angle is $44^{\circ}$, the resolution is $1.07 \mathrm{~m} \mathrm{x}$ $0.679 \mathrm{~m}$ in azimuth-slant range. Near range is on the left. 
- data types adequate to obtain images with similar characteristics (e.g. in terms of resolution).

In particular, concerning the last point, for RADARSAT-2 the Ultra Fine mode and the Fine Quad-Pol mode have been considered: the first mode will guarantee a resolution similar to the Cosmo-SkyMed Stripmap acquisition mode, while the second one will allow for a complete polarimetric characterization, although at the expense of a coarser resolution.

Finally, it is important to note that the acquisition parameters have been selected to be comparable to those of Cosmo/SkyMed data acquired in 2011 in the framework of the first Cosmo AO [7]. One example of these data is shown in Fig. 1. These acquisitions will provide the possibility to compare the results of the present project to previous results [7].

Once the first data will be obtained and after proper absolute radiometric calibration, the models described in the previous section will be tested and their potentialities for information extraction and physical parameters retrieval will be analyzed. The first results of the application of the models to actual data will be described during conference presentation.

\section{CONCLUSION}

In this paper we described the preliminary results of a projected founded in the framework of the AO regarding joint application of Cosmo/SkyMed and RADARSAT-2 data. In particular, proper scattering models for the interpretation of SAR images of urban areas have been introduced and the special issues related to the synergic use of Cosmo/SkyMed and RADARSAT-2 data for urban areas monitoring have been highlighted. Finally, relevant details regarding the ongoing data acquisition phase have been provided.

\section{ACKNOWLEDGEMENT}

This work was supported by ASI and CSA in the framework of the SOAR Cosmo/SkyMed-RADARSAT-2 joint AO project "Buildings Feature Extraction from Single SAR Images - Joint Application to COSMO-SkyMed RADARSAT-2 Images".

\section{REFERENCES}

[1] G. Franceschetti, A. Iodice, and D. Riccio, "A Canonical Problem in Electromagnetic Backscattering From Buildings," IEEE Trans. Geosci. Remote Sens., vol. 40, no. 8, pp. 1787-1801, 2002.

[2] G. Franceschetti, A. Iodice, D. Riccio, and G. Ruello, "SAR Raw Signal Simulation for Urban Structures," IEEE Trans. Geosci. Remote Sens., vol. 41, no. 9, pp. 1986-1995, 2003.

[3] R. Guida, A. Iodice, D. Riccio, and U. Stilla, "Model-Based Interpretation of High-Resolution SAR Images of Buildings," IEEE J. Sel. Top. Appl. Earth Obs. Remote Sens., vol. 1, no. 2, pp. 107$119,2008$.

[4] S. Auer, S. Hinz, and R. Bamler, "Ray-Tracing Simulation Techniques for Understanding High-Resolution SAR Images," IEEE Trans. Geosci. Remote Sens., vol. 48, no. 3, pp. 1445-1456, Mar. 2010.

[5] S. Auer and S. Gernhardt, "Linear Signatures in Urban SAR Images - Partly Misinterpreted?," IEEE Geosci. Remote Sens. Lett., vol. 11, no. 10, pp. 1762-1766, 2014.

[6] A. Ciotola, G. Di Martino, A. Iodice, D. Riccio, and G. Ruello, "Scattering Model for a Couple of Buildings in SAR Images," in Proceedings IGARSS '14, 2014, pp. 321-324.

[7] D. Riccio, G. Di Martino, G. Franceschetti, A. Iodice, A. Natale, P. Imperatore, G. Ruello, and I. Zinno, "Cosmo-SkyMed AO projects - Buildings Feature Extraction from Single SAR Images", Proceedings IGARSS 2012, Munich (Germany), July 2012, pp. 5502-5505. 